\title{
Comparative Study of Acoustical and Transport Properties of Dimethyl Sulfoxide and Acetonitrile
}

\author{
UPASNA MAGOTRA, SANDARVE and MEENA SHARMA*
}

Department of Chemistry, University of Jammu, Jammu 180 006, India

mlakhanpal89@rediffmail.com

Received 3 June 2013 / Accepted 10 July 2013

\begin{abstract}
Ultrasonic velocity, density and viscosity of different concentrations of aqueous solutions of dimethyl sulfoxide (DMSO) and acetonitrile (ACN) have been studied at 298.15 K. From the experimental data various acoustical parameters such as Free volume $V_{\mathrm{f}}$, Relaxation time $\tau$, Gibb's free energy $\Delta \mathrm{G}$, Absorption coefficient $\alpha / \mathrm{f}^{2}$ have been evaluated. Also hydrodynamic flow studies of different concentrations of experimental solvents through commercially available Acroshield ${ }^{\mathrm{TM}} \mathrm{H}$ 69008 composite cellophane membrane are described and used to evaluate transport properties such as permeability coefficient $\mathrm{L}_{\mathrm{p}}$ and frictional coefficient $\mathrm{F}_{\mathrm{wm}}$. The acoustical parameters of different concentrations of aqueous solutions of DMSO and ACN were correlated with their transport properties. The results are discussed in the light of intermolecular interactions occurring in the solutions and also in terms of interactions between membrane and solution.
\end{abstract}

Keywords: Ultrasonic velocity, Free volume, Absorption coefficient, Acroshield ${ }^{\mathrm{TM}}$ H 69008 composite cellophane membrane, Permeability coefficient and Frictional coefficient

\section{Introduction}

The study of intermolecular interaction plays an important role in the development of molecular sciences. A large number of studies have been made on the molecular interaction in liquid systems by various physical methods like Infrared, Raman effect, Nuclear Magnetic resonance, Dielectric constant, ultra violet and ultrasonic method. In recent years ultrasonic technique has become a powerful tool in providing information regarding the molecular behaviour of liquids and solids owing to its ability of characterizing physiochemical behaviour of the medium ${ }^{1}$. On the other hand study of transport properties across membrane also provides information regarding molecular interactions as well as interactions of solute and solvent with the membrane. The survey of literature shows that the data on transport properties and acoustic properties are being increasingly studied for developing the correlation between the two ${ }^{2-8}$. Many attempts are being made to formulate a relationship between these properties. It has been widely accepted that the gas diffusion 
through a polymer can be related to free volume through the Doolittle relation $\mathrm{D}=$ Aexp $(-\mathrm{B} / \mathrm{f})$, where ' $\mathrm{f}$ ' is the free volume, $\mathrm{A}$ and $\mathrm{B}$ are constants. The practical utility of the new relation is that it can be used as an efficient tool for predicting transport properties in a wide range of polymers. The present investigation deals with the study of molecular interactions in aqueous solutions of DMSO and acetonitrile (ACN) at $298.15 \mathrm{~K}$ with the help of acoustical as well as transport properties. The water + organic type solvents are frequently used as chemical and biochemical reaction media ${ }^{9}$. Hence the studied systems had industrial utility.

\section{Experimental}

DMSO (AR grade) was used as such. ACN (AR grade) after keeping over anhydrous calcium oxide for about 48 hours was shaken with phosphorus pentoxide and was distilled. Ordinary water distilled thrice over alkaline $\mathrm{KMnO}_{4}$ and acidic $\mathrm{K}_{2} \mathrm{Cr}_{2} \mathrm{O}_{7}$ in all glass apparatus was used for preparing the solutions. The specific conductance of this water was $1.30 \times 10^{-4} \mathrm{~S} \mathrm{~m}^{-1}$.

The ultrasonic velocity, ' $U$ ' of solutions was measured by determining the wavelength of sound with the help of multi frequency ultrasonic interferometer (M-82S, Mittal Enterprises, India) at $6 \mathrm{MHz}$. The temperature of water surrounding the measuring cell was controlled and accuracy in the velocity measurement was $+0.05 \% .20 \mathrm{~mL}$ specific gravity bottle was used to measure density, ' $\rho$ ' which was calibrated using triply distilled water. The specific gravity bottle containing the experimental solutions was immersed in a constant temperature bath controlled with in $+0.05 \mathrm{~K}$. The weighing was performed in an analytical balance (Mettler Toledo) having an accuracy of $1.0 \times 10^{-5} \mathrm{~g}$. The viscosity, ' $\eta$ ' was measured with Ostwald type viscometer which was precalibrated using triply distilled water. The ultrasonic velocity, density and viscosity measurements were measured at least thrice for each sample and found to be repeatable within the precision limits. The Acroshield ${ }^{\mathrm{TM}} \mathrm{H}$ 69008 composite cellophane membrane was thoroughly cleaned with distilled water, dried and weighed. The membrane was kept in the solutions of different concentrations of the two experimental liquids for $48 \mathrm{~h}$. After 48 hours, membrane was again thoroughly cleaned with distilled water, dried and weighed. The membrane was found to be stable over entire range of DMSO concentration but it disintegrated above $40 \%$ concentration of ACN. Therefore, low concentrations for preparation of solutions were chosen. The apparatus and procedure used in the present investigation for evaluating transport properties is the same as described elsewhere ${ }^{10}$.

\section{Results and Discussion}

The ultrasonic velocity measurement is extensively used to study the physicochemical behavior of liquids. With the help of measurement of density and viscosity, various acoustical parameters like free volume, relaxation time, Gibb's free energy and absorption coefficient were calculated by using the following expressions-

Free volume $\quad V_{f}=\left[M_{\text {eff }} u / K n\right]^{3 / 2}$

Where $\mathrm{M}_{\text {eff }}$ is the effective molecular weight $\left(\mathrm{M}_{\text {eff }}=\Sigma \mathrm{m}_{\mathrm{i}} \mathrm{x}_{\mathrm{i}}\right.$, in which $\mathrm{m}_{\mathrm{i}}$ and $\mathrm{x}_{\mathrm{i}}$ are the molecular weight and the mole fraction of the individual constituents respectively). $\mathrm{K}$ is a temperature independent, constant which is equal to $4.28 \times 10^{9}$ for all liquids.

Relaxation time $\quad \tau=4 / 3 \beta_{a d} \eta$

Where $\beta_{\text {ad }}$ denotes adiabatic compressibility and $\eta$ denotes viscosity. 
Gibb's free energy $\Delta G=k \operatorname{Tin}(K T \tau / h)$ where $\mathrm{k}$ is the Boltzmann constant $\left(1.38 \times 10^{-23} \mathrm{JK}^{-1}\right), \mathrm{T}$ is absolute temperature, $\mathrm{h}$ is Planck's constant $\left(6.62 \times 10^{-34} \mathrm{Js}\right)$ and $\tau$ is the relaxation time.

Absorption coefficient $\alpha / f^{2}=4 \pi^{2} \tau / 2 u$

For evaluation of transport properties, following equations were used-Hydrodynamic permeability $J V=\pi r^{2} x / \pi R^{2} T$ where ' $x$ ' is the distance moved in the capillary of the apparatus in time ' $t$ ', ' $r$ ' is the radius of the capillary and ' $R$ ' is the radius of the membrane.

Permeability coefficient $L_{p}=J v / \Delta P$ where $\Delta \mathrm{P}$ is the applied pressure difference. $\mathrm{L}_{\mathbf{p}}$ has the character of mobility and represents the velocity of the fluid per unit pressure difference for the unit cross-sectional area of membrane.

Frictional coefficient $F_{w m}=\Phi_{w} V_{w} / L_{p} \delta$ where ' $\bar{V}_{w}$ ' is the water content of the membrane and is expressed as the volume fraction of the total membrane volume, ' $\Phi_{\mathrm{w}}$ ' is numerically equal to the fraction of membrane surface available for the permeation of the solution. It was determined by the method described by Ginzberg and Katchalsky ${ }^{11}$ and the value obtained was 0.4417391 in the case of Acroshield ${ }^{\mathrm{TM}} \mathrm{H} 69008$ composite cellophane membrane, $\delta$ is the thickness of the membrane and its value in the given case is $0.42 \times 10^{-2} \mathrm{~m}, \overline{\mathrm{V}}_{\mathrm{w}}$ is the molar volume of water.

Table 1. Ultrasonic velocity, density and viscosity of aqueous solution of DMSO and ACN at $298.15 \mathrm{~K}$

\begin{tabular}{ccccccc}
\hline Conc. & \multicolumn{2}{c}{${\mathrm{U}, \mathrm{ms}^{-1}}^{-1}$} & \multicolumn{2}{c}{$\rho \times 10^{-3}, \mathrm{~kg}^{-3}$} & \multicolumn{1}{c}{$\eta \times 10^{3}, \mathrm{Nm}^{-2} \mathrm{~s}$} \\
\hline & DMSO & ACN & DMSO & ACN & DMSO & ACN \\
\hline $10 \%$ & 1550.1818 & 1542.5454 & 1.0112 & 0.9839 & 0.9192 & 0.8052 \\
$12.5 \%$ & 1561.6363 & 1544.1817 & 1.0142 & 0.9803 & 0.9762 & 0.8195 \\
$15 \%$ & 1573.0909 & 1545.8181 & 1.0171 & 0.9767 & 1.0332 & 0.8338 \\
$17.5 \%$ & 1584.5454 & 1547.4545 & 1.0214 & 0.9721 & 1.1111 & 0.8478 \\
$20 \%$ & 1596.0000 & 1549.0909 & 1.0255 & 0.9674 & 1.1890 & 0.8617 \\
$22.5 \%$ & 1608.0000 & 1544.7273 & 1.0297 & 0.9638 & 1.2354 & 0.7778 \\
$25 \%$ & 1620.0000 & 1540.3636 & 1.0338 & 0.9602 & 1.2818 & 0.6938 \\
$27.5 \%$ & 1632.5454 & 1533.2727 & 1.0381 & 0.9562 & 1.3483 & 0.6746 \\
$30 \%$ & 1645.0909 & 1526.1818 & 1.0422 & 0.9522 & 1.4147 & 0.6554 \\
\hline
\end{tabular}

Table 2. Acoustical properties of aqueous solution of DMSO and ACN at $298.15 \mathrm{~K}$

\begin{tabular}{lcccccccc}
\hline Conc. & \multicolumn{2}{c}{$\mathrm{V}_{\mathrm{f}} \times 10^{8} \mathrm{~m}^{3} \mathrm{~mol}^{-1}$} & \multicolumn{2}{c}{$\tau \times 10^{13}(\mathrm{~s})$} & \multicolumn{2}{c}{$\Delta \mathrm{G \times 10^{22 }} \mathrm{Jmol}^{-1}$} & \multicolumn{2}{c}{$\alpha / \mathrm{f}^{2} \times 10^{15} \mathrm{~ms}^{2}$} \\
\hline & DMSO & ACN & DMSO & CAN & DMSO & ACN & DMSO & ACN \\
\hline $10 \%$ & 2.1547 & 2.4513 & 5.0435 & 4.5854 & 46.9796 & 43.0604 & 6.4156 & 5.8618 \\
$12.5 \%$ & 2.0599 & 2.4357 & 5.2625 & 4.6744 & 48.7289 & 43.8519 & 6.6451 & 5.9692 \\
$15 \%$ & 1.9811 & 2.4219 & 5.4731 & 4.7633 & 50.3428 & 44.6272 & 6.8607 & 6.0763 \\
$17.5 \%$ & 1.8613 & 2.4119 & 5.7768 & 4.8561 & 52.5666 & 45.4209 & 7.1891 & 6.1881 \\
$20 \%$ & 1.7622 & 2.4043 & 6.0689 & 4.9490 & 54.5967 & 46.2009 & 7.4985 & 6.2998 \\
$22.5 \%$ & 1.7460 & 2.8489 & 6.1867 & 4.5094 & 55.3872 & 42.3725 & 7.5869 & 5.7565 \\
$25 \%$ & 1.7351 & 3.4376 & 6.2991 & 4.0602 & 56.1282 & 38.0548 & 7.6675 & 5.1977 \\
$27.5 \%$ & 1.6907 & 3.6371 & 6.4977 & 4.0013 & 57.4054 & 37.4530 & 7.8484 & 5.1460 \\
$30 \%$ & 1.6554 & 3.8547 & 6.6874 & 3.9401 & 58.5897 & 36.8188 & 8.0160 & 5.0908 \\
\hline
\end{tabular}


Table 3. Transport properties of aqueous solution of DMSO and ACN at 298.15K.

\begin{tabular}{ccccccc}
\hline Conc. & \multicolumn{2}{c}{$\mathrm{J}_{\mathrm{v}} \times 10^{4} \mathrm{~ms}^{-1}$} & \multicolumn{2}{c}{$\mathrm{Lp} \times 10^{7} \mathrm{~m}^{3} \mathrm{~N}^{-1} \mathrm{~s}^{-1}$} & \multicolumn{2}{c}{$\mathrm{F}_{\mathrm{wm}} \times 10^{-9} \mathrm{mNmol}^{-1} \mathrm{~s}$} \\
\hline & DMSO & ACN & DMSO & ACN & DMSO & ACN \\
\hline $10 \%$ & 18.0621 & 19.5627 & 7.2248 & 7.8251 & 2.6204 & 2.4194 \\
$12.5 \%$ & 17.5514 & 19.2926 & 7.0206 & 7.7170 & 2.6966 & 2.4532 \\
$15 \%$ & 16.9723 & 19.0732 & 6.7889 & 7.6293 & 2.7886 & 2.4814 \\
$17.5 \%$ & 16.3951 & 17.6345 & 6.5580 & 7.0538 & 2.8868 & 2.6839 \\
$20 \%$ & 15.8532 & 16.1251 & 6.3413 & 6.4500 & 2.9855 & 2.9351 \\
$22.5 \%$ & 15.7145 & 14.1623 & 6.2858 & 5.6649 & 3.0118 & 3.3419 \\
$25 \%$ & 15.5332 & 12.2714 & 6.2133 & 4.9086 & 3.0469 & 3.8568 \\
$27.5 \%$ & 15.3226 & 11.7921 & 6.1290 & 4.7168 & 3.0889 & 4.0137 \\
$30 \%$ & 15.1527 & 11.2519 & 5.0611 & 4.5008 & 3.1235 & 4.2063 \\
\hline
\end{tabular}

\section{Conclusion}

From Table 1 it was observed that viscosity of aqueous solutions of DMSO increases with rise in concentration which indicates the existence of strong interaction between solute and solvent. This is also supported by ultrasonic velocity and other acoustical parameters. However for ACN there is an increase in viscosity and ultrasonic velocity up to $20 \%$ (mole fraction 0.09), there after it decreases. This suggests formation of clathrate-like hydrates with water ${ }^{12}$ which resulted in decrease in solute-solvent interactions.

The molecules of liquid are not closely packed and as such there is always some free space between them. This free space is known as free volume. It is observed that free volume for aqueous solution of DMSO decreases with rise in concentration as shown in Table 2 which shows that solute solvent molecules are coming close to each other and the space between them is decreasing with rise in concentration. This supports to the strong solute-solvent interaction in DMSO solution. However for aqueous solution of ACN a decrease in free volume was observed up to $20 \%$, there after it increases which again supports the formation of clathrate-like hydrates of ACN with water. Formation of clathratelike hydrates by ACN with water is also supported by other acoustical parameters such as relaxation time, Gibb's free energy and absorption coefficient. From Table 3 it was also observed that hydrodynamic permeability and permeability coefficient decreases with increase in concentration for both the experimental solvent solutions. These results are in accordance with the fact that permeability is inversely proportional to viscosity ${ }^{13}$. Also it is observed that values of hydrodynamic permeability and permeability coefficient for aqueous solution of ACN were greater than aqueous solution of DMSO up to $20 \%$, thereafter trend was reversed. This is again due to formation of clathrate-like hydrates of ACN with water. As the pore size of the membrane was small, formation of large sized hydrates by ACN resulted in decrease in permeability of the membrane beyond $20 \%$ concentration of ACN as compared to DMSO. Values of frictional coefficient also support the same result. Hence, both thermoacoustical parameters and transport properties support the formation of clathrate-like hydrates by ACN with water.

\section{References}

1. Priya C S, Nithya S, Velraj G and Kanappan A N, Int J Adv Sci Tech., 2010, 18, 59.

2. Blokhra R L and Awasthi A, Indian J Pure Appl Phys., 1992, 30(12), 760-763.

3. Blokhra R L and Nag A, Indian J Pure Appl Phys., 1991, 29, 756.

4. Thornton A W, Nairn K M, Hill J A and Hill J M, J Membr Sci., 2009, 338(1-2), 29-37; DOI:10.1016/j.memsci.2009.03.053 
5. Thornton A W, Nairn K M, Hill A J, Hill J M and Huang Y, J Membr Sci., 2009, 338(1-2), 38-42; DOI:10.1016/j.memsci.2009.03.057

6. Mondal S, Hu J L and Yong Z, J Membr Sci., 2006, 280(1-2), 427-432; DOI:10.1016/j.memsci.2006.01.047

7. Gedde W U, Larson P T, Edsberg L, Angelstok A and Hedenqvist M, Polymer, 1996, 37(14), 2887-2902; DOI:10.1016/0032-3861(96)89384-0

8. Wijmans J G, J Membr Sci., 2004, 237(1-2), 39-50 ; DOI:10.1016/j.memsci.2004.02.028

9. Iulian O and Ciocirlan O, Rev Roum Chim., 2010, 55, 45-53.

10. Sharma M, Sharma A K and Lal R, Res J Chem Environ., 2010, 14(3), 75.

11. Ginzberg B Z and Katchalsky A, J Gen Physiol., 1963, 47(2), 403-418.

12. Jerie K, Baranowski A, Koziol S, Glinski J and Burakowski A, Chem Phys., 2005, 309(2-3), 277-282; DOI:10.1016/j.chemphys.2004.09.015

13. Kesting R E, Subcasky W J and Patton J D, J Colloid Interface Sci., 1968, 28(1), 156-160; DOI:10.1016/0021-9797(68)90217-8 